\title{
Integrated single cell data analysis reveals cell specific networks and novel coactivation markers
}

\author{
Shila Ghazanfar ${ }^{*}$, Adam J. Bisogni ${ }^{2}$, John T. Ormerod ${ }^{1,3}$, David M. Lin² and Jean Y. H. Yang \\ From 15th International Conference On Bioinformatics (INCOB 2016) \\ Queenstown, Singapore. 21-23 September 2016
}

\begin{abstract}
Background: Large scale single cell transcriptome profiling has exploded in recent years and has enabled unprecedented insight into the behavior of individual cells. Identifying genes with high levels of expression using data from single cell RNA sequencing can be useful to characterize very active genes and cells in which this occurs. In particular single cell RNA-Seq allows for cell-specific characterization of high gene expression, as well as gene coexpression.

Results: We offer a versatile modeling framework to identify transcriptional states as well as structures of coactivation for different neuronal cell types across multiple datasets. We employed a gamma-normal mixture model to identify active gene expression across cells, and used these to characterize markers for olfactory sensory neuron cell maturity, and to build cell-specific coactivation networks. We found that combined analysis of multiple datasets results in more known maturity markers being identified, as well as pointing towards some novel genes that may be involved in neuronal maturation. We also observed that the cell-specific coactivation networks of mature neurons tended to have a higher centralization network measure than immature neurons.

Conclusion: Integration of multiple datasets promises to bring about more statistical power to identify genes and patterns of interest. We found that transforming the data into active and inactive gene states allowed for more direct comparison of datasets, leading to identification of maturity marker genes and cell-specific network observations, taking into account the unique characteristics of single cell transcriptomics data.
\end{abstract}

Keywords: Single-cell transcriptomics, RNA-sequencing, Mixture modelling, ScRNA-Seq, Olfactory sensory neuron, Neuron

\section{Background}

High throughput transcriptome profiling of single cells has exploded in recent years in the areas of biomedical and basic science research. Single cell RNA-Seq (scRNASeq) has been employed to study many types of cells in a number of organisms, including stem cells, cancer cells and neurons in mouse and human [1]. This technology has enabled both small-scale interrogations (16

${ }^{*}$ Correspondence: shila.ghazanfar@sydney.edu.au

'School of Mathematics and Statistics, The University of Sydney, Eastern Avenue, Camperdown, NSW, 2006, Australia

Full list of author information is available at the end of the article cells [2]) to very large scale profiling studies $(44,808$ cells [3]) on a transcriptome level. Analysis approaches have aimed to characterize cell heterogeneity, and to identify subtypes using techniques such as dimension reduction and clustering. Other possible analyses include differential expression, and interrogating more specific questions. For instance, a long standing hypothesis has been that olfactory sensory neurons (OSNs) in mice express only one odorant receptor gene, termed the 'one-neuron-onereceptor' rule, which was able to be tested in single mature and immature OSNs through scRNA-Seq, and the authors found that immature neurons can transiently express multiple odorant receptor genes [4] while mature neurons 
primarily express one odorant receptor gene. A number of tools and approaches have emerged recently offering extensive pipelines from raw reads to analysis results [5-7], and others that focus on particular aspects of a typical scRNA-Seq analysis, such as clustering $[8,9]$ and differential expression analysis [10].

Some statistical challenges associated with scRNA-Seq are unique compared to typical RNA-Seq of bulk cell populations. While shared challenges such as normalization, accurate modeling of counts and cross platform comparisons exist, these may be exacerbated or manifest differently in the presence of features unique to scRNA-Seq data. The most immediate characteristic of single cell gene expression count matrices are that there is an abundance of zeros, i.e. genes with no read counts [10], that persist even after transformations such as counts per million (CPM) or reads per kilobase per million reads (RPKM). Furthermore, the proportion of zeros across genes appears to be related to the depth of sequencing performed, contributing to the challenge of appropriately comparing between multiple datasets with different levels of read depths achieved.

Another key aspect of scRNA-Seq data is the apparent bimodality of non-zero expression values [11-13]. As this phenomenon is also observed in other single-cell gene expression measurement methods such as fluorescence in situ hybridization (FISH) [11], it is believed that this phenomenon is not attributed to technical artifacts stemming from the scRNA-Seq experiments. Rather, examining the distribution of gene expression measurements of a given gene over many cells can uncover three distinct transcriptional states: no expression, characterized by no observed read counts; low expression, where RNA is present at a low level and possibly undergoing degradation; and high expression, where RNA may have been produced through a 'bursting' process [14]. Existing approaches for classifying cells into a low or high expression state are few, including imposing a strict threshold value, and fitting Gaussian mixture models [15].

To this end, in this manuscript we offer a versatile modeling framework to identify transcriptional states as well as structures of coactivation for different neuronal cell types across multiple datasets. This framework includes (1) a gamma-normal mixture modeling approach to classify each gene into no, low or high expression within each cell; (2) the identification of coactivation networks within each cell and (3) creation of a uniqueness metric to identify cell type specific genes across multiple scRNA-Seq datasets. Furthermore, we focused on three specific datasets that studied olfactory sensory neurons, and discovered that the topology of coactivation networks of each cell changes as the olfactory sensory neuron cells mature. This work enables discovery of biologically meaningful genes through combined analysis of coactivation with genes known to be related to neuron maturity.

\section{Methods}

\section{Data collection and preprocessing}

A set of nine single cell RNA-Seq datasets were curated (Table 1), all measuring transcriptomes of various neuronal cell populations in mice, with varying numbers of cells, sequencing strategies, and overall read depths. Raw sequencing reads were downloaded from the Gene Expression Omnibus (GEO), the Sequence Read Archive (SRA) or the European Nucleotide Archive (ENA). Fastq files were each mapped to the $\mathrm{mm} 10$ reference genome using STAR RNA-Seq aligner [16] with default parameters. The resulting mapped read files were then converted

Table 1 Description of nine murine neuronal single-cell RNA-Seq datasets. DRG - dorsal root ganglion

\begin{tabular}{|c|c|c|c|c|c|}
\hline Author & $\begin{array}{l}\text { GEO/SRA } \\
\text { Accession }\end{array}$ & $\begin{array}{l}\text { Number } \\
\text { of Cells }\end{array}$ & Cell Type(s) & $\begin{array}{l}\text { Read } \\
\text { Length }\end{array}$ & $\begin{array}{l}\text { Median Read } \\
\text { Depth }\end{array}$ \\
\hline Fuzik et al. [37] & GSE70844 & 83 & Excitatory pyramidal and inhibitory neurons & 51 & 391,449 \\
\hline Hanchate et al. [25] & GSE75413 & 93 & Olfactory sensory neurons & 98 & $3,352,691$ \\
\hline Li et al. [23] & GSE63576 & 209 & Somatosensory DRG neurons & 200 & $18,300,045$ \\
\hline Lovatt et al. [24] & GSE52525 & 28 & $\begin{array}{l}\text { Mixed cultures of dispersed braincells, hippocam- } \\
\text { pal pyramidal neurons }\end{array}$ & 202 & $17,727,180$ \\
\hline Saraiva et al. [15] & $\begin{array}{l}\text { PRJEB4014, } \\
\text { PRJEB8101, } \\
\text { PRJEB4461 }\end{array}$ & 264 & Olfactory sensory neurons & 200 & $1,570,234$ \\
\hline Tan et al. [4] & SRP065920 & 143 & Olfactory sensory neurons & 100 & 936,016 \\
\hline Tasic et al. [38] & GSE71585 & 1,809 & Cortical cells & 89 & $2,350,114$ \\
\hline Usoskin et al. [39] & GSE59739 & 864 & Lumbar DRG neurons & 40 & 86,588 \\
\hline Zeisel et al. [40] & GSE60361 & 3,005 & Somatosensory and hippocampal C1 neurons & 52 & 496,431 \\
\hline Total & & 6498 & & & \\
\hline
\end{tabular}


to bam, sorted and indexed using Samtools [17], and read counts for a total of 38806 genes were obtained using HTSeq-count [18] under the mode 'union' with other default parameters. Read counts for multiple runs belonging to the same cell were added together, resulting in a raw count matrix for 38806 genes and 6377 cells. The data matrix was further transformed by calculating counts per million mapped reads (CPM) and taking the shifted $\log (\log 2 \mathrm{CPM})$, i.e. $y_{i j}=\log _{2}\left(1+10^{6} r_{i j} / \sum_{k} r_{k j}\right)$, where $r_{i j}$ are the raw read counts and $y_{i j}$ the transformed counts for gene $i$ and cell $j$. Following this, we fitted gamma-normal mixture models per gene per dataset, initially removing cells with zero $\log 2 \mathrm{CPM}$ values, as described in the next section.

\section{Gamma-normal mixture modeling}

To model the distribution of gene expression values, we considered a gamma-normal mixture model. The gamma distribution is fairly flexible with two parameters, and takes non-negative values. We observe that scRNA-Seq gene expression values on $\log 2 \mathrm{CPM}$ scale also take nonnegative values and thus this distribution may be suitable. We may be able to use a simpler distribution with similar properties, such as the exponential distribution, however this does not appear to be as flexible as desired. As well as this, the normal distribution is a suitable candidate for the second component of the mixture model as it is fairly well characterized.

Before continuing with fitting the gamma-normal mixture model, we remove the zeroes from the data, as data arising from scRNA-Seq exhibits an extremely high proportion of genes and cells with zero counts, resulting in ill-fitting model parameters.

The remainder of this section derives the expectation maximization (EM) algorithm for fitting the gammanormal mixture model. We assume that for a given gene, the non-zero expression values can be described by a mixture of gamma and normal distributions, where the gamma component corresponds to lowly expressed cells and the normal components corresponds to the highly expressed cells. Let $\mathbf{x}=\left(x_{1}, x_{2}, \ldots, x_{n}\right)$ be a vector of non-zero $\log 2 \mathrm{CPM}$ expression values for a given gene. The density functions for the gamma and normal component are

$$
\begin{aligned}
f(w, \alpha, \beta) & =\frac{\beta^{\alpha}}{\Gamma(\alpha)} w^{\alpha-1} e^{-\beta w}, \quad \text { and } \\
f\left(w, \mu, \sigma^{2}\right) & =\frac{1}{\sigma \sqrt{2 \pi}} e^{-\frac{(w-\mu)^{2}}{2 \sigma^{2}}}
\end{aligned}
$$

respectively. Let $\mathbf{y}=\left(y_{1}, y_{2}, \ldots, y_{n}\right)$ be a binary vector indicating membership of each cell in the normal component of the mixture. We assume that $y_{i}$ is generated from an independent Bernoulli distribution with probability of success $\rho, y_{i} \sim B(1, \rho)$ for $i=1,2, \ldots, n$.
Thus the density function for $x_{i}$ is

$$
\begin{aligned}
f\left(x_{i}, \alpha, \beta, \mu, \sigma^{2}, \rho\right)= & (1-\rho) \frac{\beta^{\alpha}}{\Gamma(\alpha)} x_{i}^{\alpha-1} e^{-\beta x_{i}} \\
& +\rho \frac{1}{\sigma \sqrt{2 \pi}} e^{-\frac{\left(x_{i}-\mu\right)^{2}}{2 \sigma^{2}}} .
\end{aligned}
$$

The corresponding complete log-likelihood is

$$
\begin{aligned}
& \ell\left(\mathbf{x}, \mathbf{y}, \alpha, \beta, \mu, \sigma^{2}, \rho\right) \\
& =\sum_{i=1}^{n}\left[\left(1-y_{i}\right)(\alpha \log (\beta)-\log \Gamma(\alpha)\right. \\
& \left.\quad+(\alpha-1) \log \left(x_{i}\right)-\beta x_{i}\right) \\
& \quad+y_{i}\left(-\frac{1}{2} \log \left(2 \pi \sigma^{2}\right)-\frac{\left(x_{i}-\mu\right)^{2}}{2 \sigma^{2}}\right)+y_{i} \log \rho \\
& \left.\quad+\left(1-y_{i}\right) \log (1-\rho)\right] .
\end{aligned}
$$

Let $z_{i}=\mathbb{E}\left(y_{i} \mid\right.$ rest $), i=1,2, \ldots, n$ be the expectation of $y_{i}$ given the other parameters and data. We also let $Q\left(\alpha, \beta, \mu, \sigma^{2}, \rho\right) \equiv \mathbb{E}\left(\ell\left(\mathbf{x}, \alpha, \beta, \mu, \sigma^{2}, \rho\right) \mid\right.$ rest $)$ be the expectation of the log-likelihood given the other parameters and data. In particular

$$
\begin{aligned}
& Q\left(\alpha, \beta, \mu, \sigma^{2}, \rho\right) \\
& =\sum_{i=1}^{n}\left[\left(1-z_{i}\right)(\alpha \log (\beta)-\log \Gamma(\alpha)\right. \\
& \left.\quad+(\alpha-1) \log \left(x_{i}\right)-\beta x_{i}\right) \\
& \quad+z_{i}\left(-\frac{1}{2} \log \left(2 \pi \sigma^{2}\right)-\frac{\left(x_{i}-\mu\right)^{2}}{2 \sigma^{2}}\right) \\
& \left.\quad+z_{i} \log \rho+\left(1-z_{i}\right) \log (1-\rho)\right] .
\end{aligned}
$$

and $z_{i}=1 /\left(1+e^{-\eta_{i}}\right)$, for $i=1,2, \ldots, n$ where $\eta_{i}$ is given by

$$
\begin{aligned}
\eta_{i}=\widehat{\alpha} & \log (\widehat{\beta})-\log \Gamma(\widehat{\alpha})+(\widehat{\alpha}-1) \log \left(x_{i}\right)-\widehat{\beta} x_{i} \\
& +\frac{1}{2} \log \left(2 \pi \widehat{\sigma}^{2}\right)+\frac{\left(x_{i}-\widehat{\mu}\right)^{2}}{2 \widehat{\sigma}^{2}}+\log \left(\frac{\widehat{\rho}}{1-\widehat{\rho}}\right) .
\end{aligned}
$$

The above describes the expectation step, while the following parameter updates describe the M-step,

$$
\begin{gathered}
\widehat{\mu}=\frac{\sum z_{i} x_{i}}{\sum z_{i}}, \quad \widehat{\sigma}^{2}=\frac{\sum z_{i}\left(x_{i}-\widehat{\mu}\right)^{2}}{\sum z_{i}}, \\
\widehat{\alpha}=\operatorname{igamma}\left(\frac{\sum\left(\log \widehat{\beta}+\log x_{i}\right)\left(1-z_{i}\right)}{\sum\left(1-z_{i}\right)}\right), \quad \widehat{\beta}=\frac{\widehat{\alpha} \sum\left(1-z_{i}\right)}{\sum x_{i}\left(1-z_{i}\right)}, \\
\text { and } \quad \widehat{\rho}=\frac{\sum z_{i}}{n} .
\end{gathered}
$$

where igamma is the inverse gamma function, implemented in $\mathrm{R}$ within the package distr. The EM updates, as indicated by hat symbol, are made until there is negligible change in the parameter updates, or until a maximum number of iterations is reached. Initial values of $z_{i}, i=1,2, \ldots, n$ are made by randomly generating from $n$ independent $B(1,0.5)$ distributions. After the algorithm converges, cell $i$ is called "highly expressed" if $z_{i} \geq 0.5$ and "lowly expressed" otherwise. 
This mixture modeling framework was applied to each single cell RNA-Seq dataset separately. The result is a ternary matrix, containing values 0 (no expression), 1 (low expression) and 2 (high expression), and NA (missing values) with the same number of rows and columns as the $\log 2$ CPM matrix. For each dataset, cell $i$ and gene $j$ the entries of the ternary matrix is

$$
a_{i j}= \begin{cases}0, & \text { if } y_{i j}=0 \\ 1, & \text { if cell } i \text { classified to gamma component for gene } j \\ 2, & \text { if cell } i \text { classified to normal component for gene } j \\ \text { NA, } & \text { if } y_{i j}>0 \quad \text { but not enough cells to fit model for gene } j\end{cases}
$$

\section{Contextualizing genes to improve mixture modeling}

We considered that there would be a large number of genes for which only a few cells have non-zero log2CPM values, rendering accurate fitting of the gamma-normal mixture models difficult. To ameliorate this issue we incorporated $\log 2 \mathrm{CPM}$ values of ten other randomly selected genes and performed the EM algorithm. This was repeated ten times for each gene, and the majority ternary value of the ten repetitions taken as the final ternary value. Ties were dealt with in a conservative manner, that is, that the smaller value was chosen as the final ternary value for that gene and cell in the case of a tie.

\section{Curating an olfactory gene list}

In order to further interrogate the data for biological relevance, we curated a set of genes of interest using Gene Ontology (GO) using the R packages GO.db v3.2.2 and org.Mm.eg.db v3.2.3. GO terms were queried using the search term "olfa", resulting in a set of 33 terms related to olfactory processes such as 'olfactory receptor activity', and a set of 1129 genes that belong to these GO terms.

\section{Identifying transcriptionally active and coactive genes}

We supposed that genes with a higher level of expression in given cells are in an active state, and thus warranted further examination. We determined that genes were 'active' in cells if they were classified into the normal component of the mixture model. We also wanted to characterize which genes tended to be in this 'active' state together for cells, i.e. coactive. In particular we generated a coactivation matrix given by $b_{i\{j k\}}=1\left\{a_{i j}=2, a_{i k}=2\right\}$ for $i=1,2, \ldots n_{g}, j=1,2, \ldots, n_{g}$, and $k=1,2, \ldots, n, n_{g}$ the number of genes and $n$ the number of cells. Following this we could aim to identify what coactive pairs of genes were common with known markers of cell types.

\section{Identifying coactivation with known maturity markers}

Next we aimed to understand which genes are markers for maturity of olfactory sensory neurons. A number of transcriptional markers are known for cell maturity and immaturity, such as $O M P$ and GAP43, respectively
[4, 18-21]. Using our estimates of transcriptionally active genes and cells, we considered coactivation of genes with these markers. We restricted cells to those that were active for $O M P$ and not for GAP43 as mature cells, and those active for GAP43 and not for OMP as immature cells, and tested for coactivation among all genes in the transcriptome via Fisher's exact test. Genes with Bonferronicorrected $P$-values below 0.01 were considered as significantly coactivated with either OMP or GAP43.

By way of evaluation of these identified marker genes, we curated lists of genes that have previous evidence as markers for mature or immature OSNs. We used a set of 8 mature-specific and 10 immature-specific marker genes from Tan et al. [4] and a set of 691 mature-specific and 847 immature-specific marker genes from Nickell et al. [22], resulting in a combined list of 692 mature-specific and 851 immature-specific marker gene names, taking into account that multiple gene name aliases may exist. Also we note that this list of identified marker genes is not exhaustive and there may be other genes that are not captured in this curated list.

\section{Weighting coactive gene networks per cell by uniqueness}

Next we attempted to better understand the variation of combinations of pairs of genes simultaneously expressed among the cells. In particular we wanted to study what gene pairs were uniquely coactive among the cells, distinguishing it from the overall population of cells. We did this by initially building gene-gene networks for each cell, taking the fully connected network of coactive genes. The number of nodes in this network is equal to the number of active genes for that cell $N$, and the number of edges is $\left(\begin{array}{c}N \\ 2\end{array}\right)$. In order to extract biologically meaningful characteristics we next incorporated a weighting per edge that took into account how often the edge was observed among the entire set of cell networks. An edge was removed if it prevalent, that is, if it was observed in more than $1 \%$ of the population of cells, resulting in a network of edges that were more uniquely coactive in that cell compared to the cell population. To ensure the robustness of the network characteristics observed, we also perturbed the threshold for prevalent edges, testing for $0.5,1,2,3,4$, and $5 \%$.

\section{Results}

Gamma-normal mixture is versatile for a number of transcriptional profiles

We found that using a gamma-normal mixture model was suitable for accommodating the different empirical densities of the neuronal scRNA-Seq data. Figure 1 shows histograms of $\log 2 \mathrm{CPM}$ values for all genes and cells for each dataset, with zeros removed. We found that while some datasets tended to have lower percentage of zeros (e.g. Li et al. [23] and Lovatt et al. [24]) resulting in a peak 


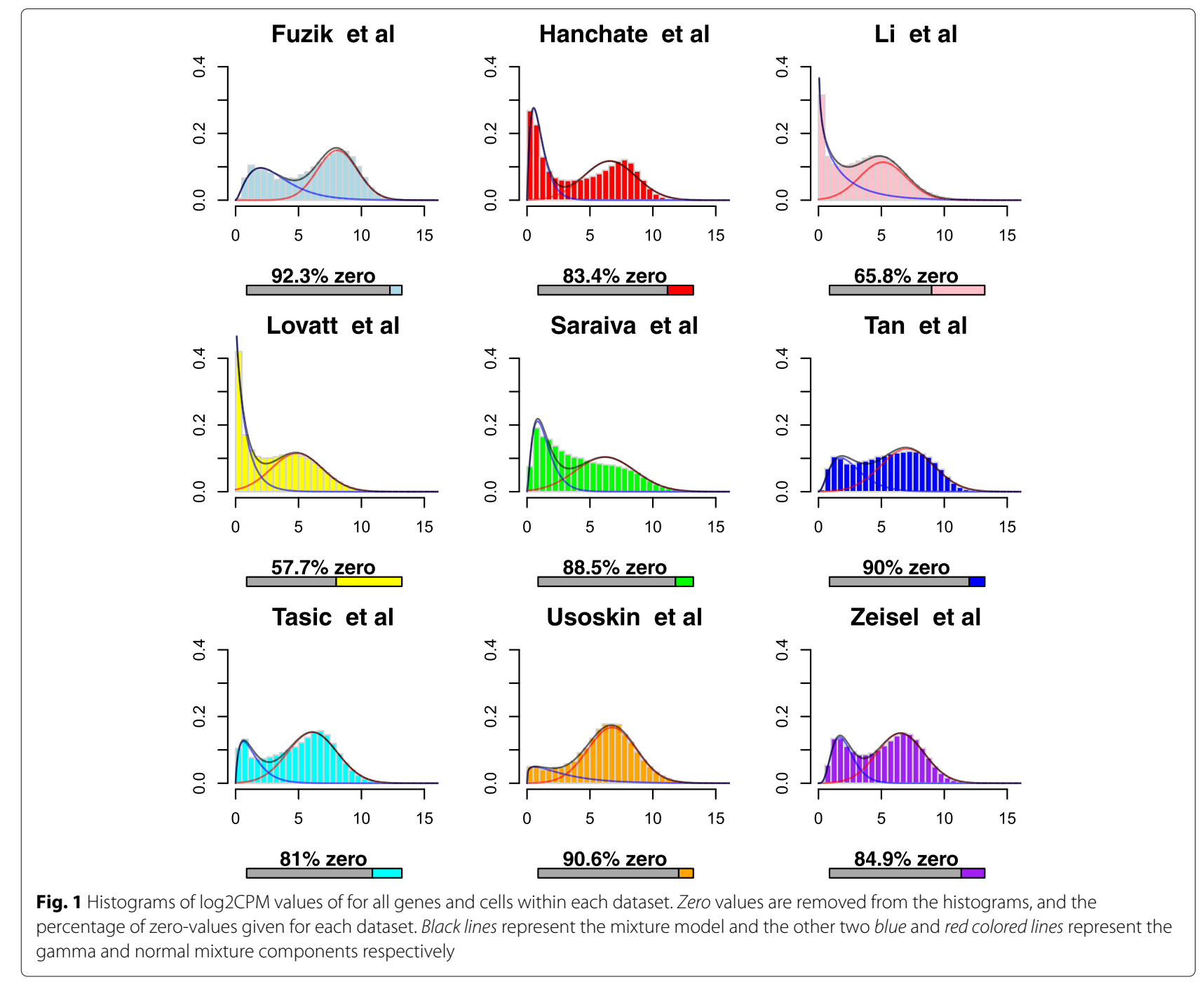

close to zero, the gamma-normal model was able to fit even this aspect of the data well.

However, since genes can have different dynamic ranges due to various technical effects (e.g. amplification or GC content bias), it is more suitable to estimate parameters of the gamma-normal mixture on a per-gene basis. Figure 2 shows histograms of $\log 2 \mathrm{CPM}$ values for genes $A C T B$, NCAM2, ACSM4, NRP1, OLFR726, for datasets Hanchate et al. [25], Saraiva et al. [15] and Tan et al. [4], as well as the estimated gamma-normal mixture model densities. These three datasets were chosen as they all profile olfactory sensory neurons (OSNs), allowing for more direct comparisons without having to account for specific cell-type differences. The modeling framework identifies when the gene is highly expressed for all cells $(A C T B$ a known housekeeping gene), as well as reasonable estimates for mixtures of lowly and highly expressed genes. However when there are too few cells with non-zero log2CPM values then the modeling framework can break down, for example the gene OLFR726 for Tan et al. [4] there are only 2 cells with non-zero $\log 2 \mathrm{CPM}$ values. We found that contextualizing genes enabled for these cells to be classified more accurately by including more data points into the mixture model. Contextualizing genes resulted in removal of missing values due to too few data points and further increased the difference between $\log 2 \mathrm{CPM}$ values for genes and cells classified as 1 (lowly expressed) and 2 (highly expressed) (Additional file 1).

\section{Incorporating ternary data slightly improves read depth} effects within datasets and facilitates clustering of cells Next we considered what impact the total depth of sequencing had on the detection of genes. We found that in general as read depth tends to increase, the number of non-zero count genes also tends to increase (Additional file 2), however it seems that this effect is strongest when read depth is relatively low. This is important since different datasets (e.g. Usoskin et al.) have a very large dynamic 


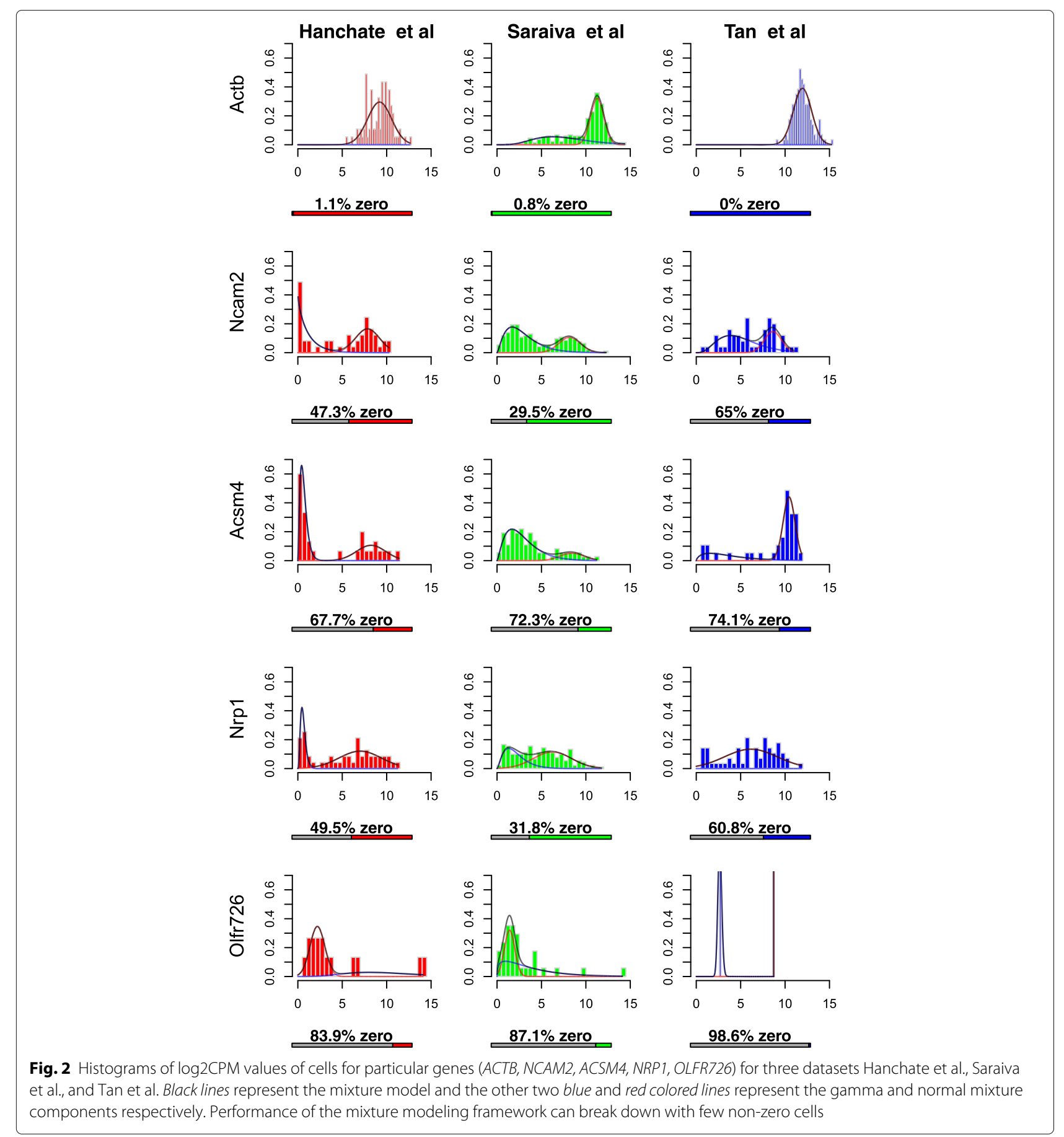

range along the total read depth of the cells, and thus the number of identified genes would be biased. This also hints towards how deeply one should sequence the mRNA within a cell to be confident of capturing enough read counts for the data to be of further use in the analysis. We found after generating ternary matrices by fitting gene-wise gamma-normal mixture models, and considering the set of genes related to olfactory GO terms that this observed relationship between read depth and number of highly expressed genes was slightly diminished (Fig. 3). However the effect of read depth and number of active genes persists for some datasets, most notably that related to Usoskin et al. Additional file 3 displays the number of non-zero count genes against number of active genes, showing that the largest change occurs with data from Lovatt et al., indicated by the fitted line. 

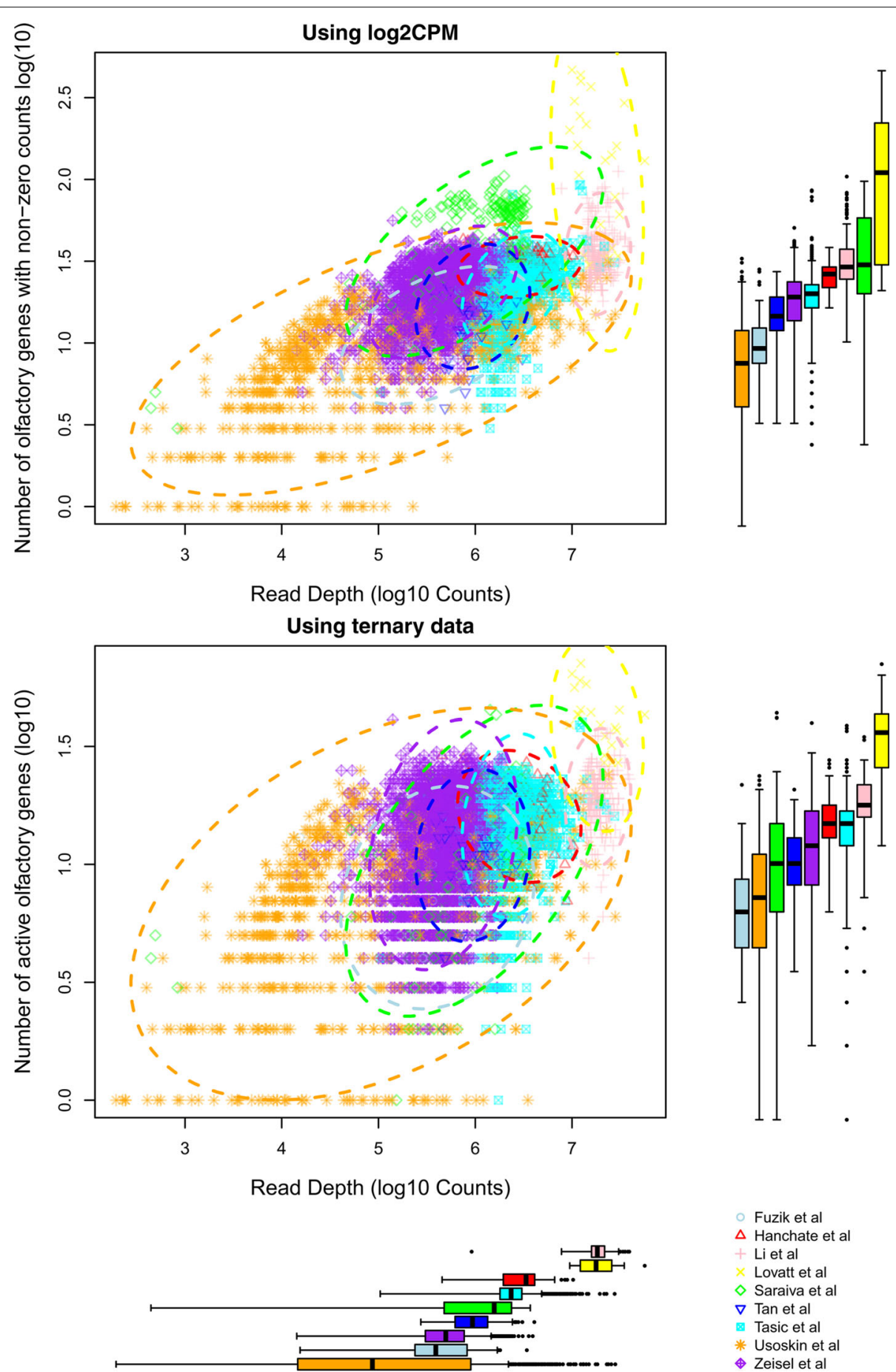

Fig. 3 Scatterplots of total read depth versus number of non-zero log2CPM values (top left) and (middle left) number of active genes using genes related to olfactory system. Boxplots (top right, middle right, respectively) are of the number of non-zero log2CPM values and number of active genes using genes related to the olfactory system respectively, split by dataset. The last boxplot (bottom left) is of total read depth of cells from various datasets. We observe some relationship between total read depth and number of non-zero genes (top left), which is slightly diminished when comparing total read depth to the number of active genes (middle left) for datasets with lower total read depth

From this point on we focused on the olfactory sensory neuron datasets Hanchate et al., Saraiva et al. and Tan et al., and on genes related to the olfactory system as curated from GO, as this allowed us to combine and analyze data sets within the context of consistent cell types. We removed cells from the Saraiva et al. dataset 
that were removed in the original analysis, due to various technical effects such as cell clumping or breakage of cells [15]. Our interest lies in only active genes, so we converted the ternary matrices to binary matrices by setting values of 0 or 1 as 0 , and values of 2 as 1 . Thus the binary matrix represented 0 for no or low expression state, and 1 for a high or active expression state. In order to ensure that this data transformation led to increased comparability, or effective standardization, of the three transformed datasets, we compared the binary matrix to the corresponding matrix of $\log 2 \mathrm{CPM}$ values in terms of classification performance. Figure 4 (left) shows the principal components analysis (PCA) for both the binary and continuous data, and we observe greater overlap of cells among the binary data than the continuous data. The hierarchical clustered heatmap of binary values in Fig. 4 (right) shows the cells, colored by dataset, are well mixed between datasets. In order to quantify what we observe in the figures, we considered how cells can be attributed to their original dataset via k-nearest neighbors

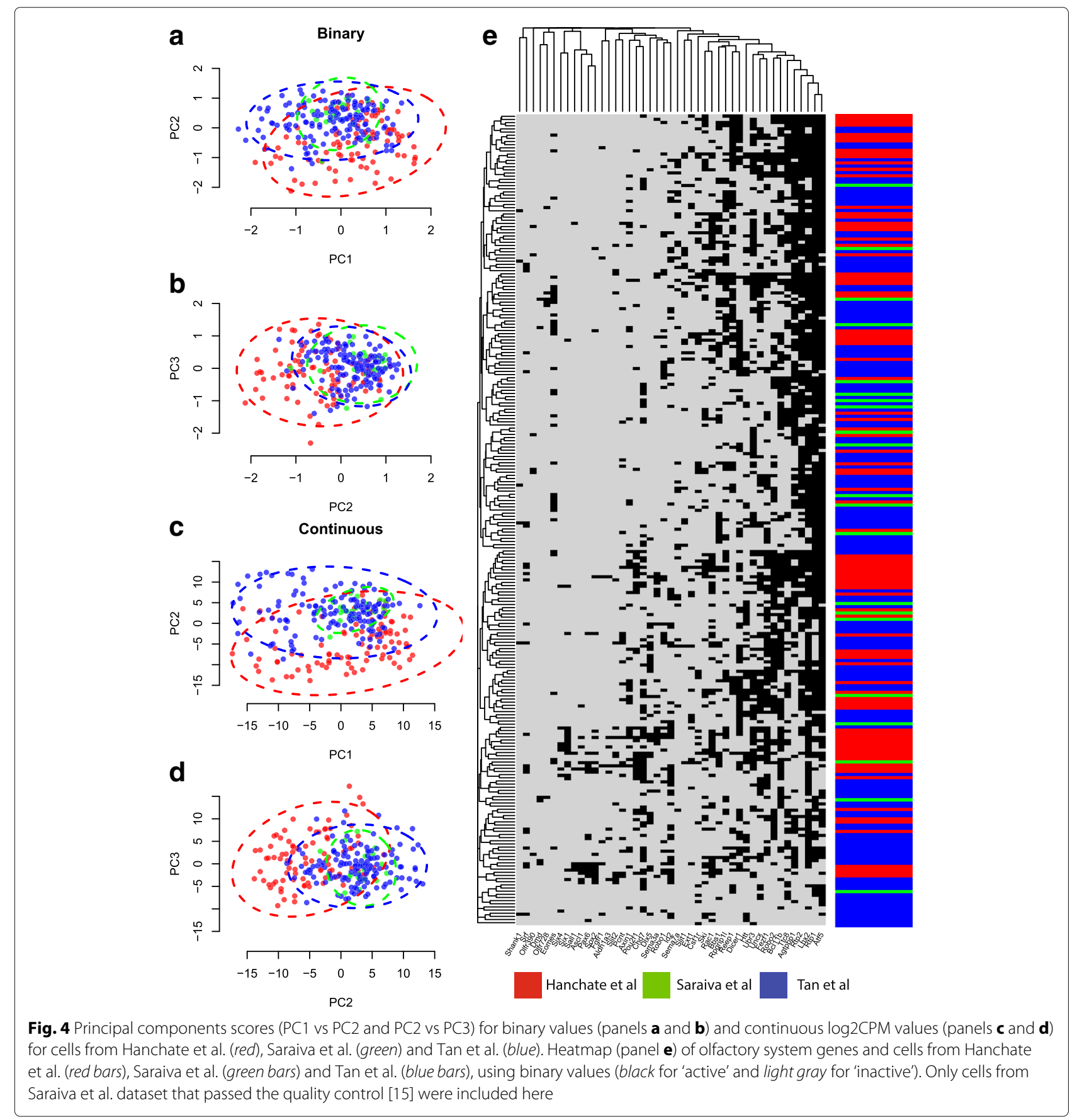


classification. Since these cells belong to the same celltype, we can assume that differences between these cells arise from non-biological factors such as technical differences. Thus if we observe a diminished classification accuracy of a cell to its original dataset label, then we can conclude that the transformation of the data results in increased comparability of the cells across the individual datasets. Indeed, performing a k-nearest neighbors classification on the originating dataset, the leave-oneout cross validation accuracy is diminished for the binary data, $66.7 \%$, than the continuous, $71.4 \%$, further assuring us that dataset specific effects are largely removed by transforming the data into binary active/non-active states.

\section{Investigating coactivation with known maturity markers recovers other known markers and integrating datasets reveals new putative markers for cell maturity}

We tested for coactivation, that is, simultaneous active states, of genes between mature and immature cells in three cases involving OSN datasets. We defined immature OSNs as those cells that were active for the gene GAP43 and inactive for $O M P$, and mature OSNs as those cells that were active for $O M P$ and inactive for GAP43, as these genes have been previously described as transcriptional markers for cell maturity and immaturity respectively [18-21]. Cells that were either active for both GAP43 and $O M P$ or not active for either were not included for further testing of coactivation. We tested for coactivation of genes to these cell combinations using Fisher's Exact Test, taking note of the gene in which coactivation occurred (coactivating with $O M P$ suggests a mature marker or coactivating with GAP43 suggests immature marker), thereby identifying if the tested gene was related positively toward maturity of immaturity. We applied this test in three cases: separately to the Hanchate et al. dataset and Tan et al. dataset, and to the concatenated dataset of Tan et al. and Hanchate et al. Note that we did not further consider the Saraiva et al. dataset as their experimental protocol selected for only mature neurons, that is those cells expressing $O M P$.

By way of evaluation, we compared the lists of putative markers for these three cases using a reference list of OSN mature and immature marker genes from Nickell et al. [22] and Tan et al. [4], a list of 692 mature genes, and 851 immature genes. This list of genes stems from literature-based and gene expression analysis and we note it may not be exhaustive, thus cannot be treated as a goldstandard positive set of genes. Comparison of our analyses of the two individual and merged datasets with the 'reference' gene list showed 95 of the 152 (62.5\%) Tan et al., 27 of the 34 (79.4\%) Hanchate et al., and 149 of the 245 (60.8\%) merged marker genes appeared in the reference mature list, and 45 of the $73(61.6 \%)$ Tan et al., 11 of the 27 (40.7\%) Hanchate et al., and 63 of the 120 (52.5\%) merged marker genes appeared in with the reference immature list.

Our analysis of the merged data sets identified 40 candidate genes that co-activated with $O M P$ but were not found to coactivate with $O M P$ when the individual datasets were analyzed alone (Table 2 and Additional file 4). Of these 40 genes, three (RTP1, RTP2, PDLIM1) are expressed in mature OSNs [26, 27]. RTP1 and RTP2 encode for proteins that facilitate the transport of odorant receptors to the membrane surface, a critical component for functional maturation of OSNs. The function of PDLIM1 in mature OSNs is unknown.

NXPH3 has been shown to be expressed in OSNs in a cAMP dependent manner [28]. Of the remaining 36 genes, none have been studied in the olfactory system. However, four are involved in ciliogenesis (CCDC114), synapse formation (NAPA), and excitation (CACNA1H, $C A R 2$ ), consistent with a role in later stages of neuronal development. An additional seven have been shown to regulate axon guidance ( $A R H G E F 28, B O C$ ), neurite outgrowth (SDC3), neuronal morphology (TPM3), and differentiation (NFATC1, CTSB, CEND1). No clear association with neuronal specific function or expression could be easily inferred for the remaining 25 genes (Additional file 4), however, none are known markers for neural immaturity. Our findings support the utility of the merged mixed model approach for enhancing the detection of coactivated genes with merged scRNA-Seq data sets. Our approach identified 40 potential new markers, at least three of which are already known to be expressed in mature OSNs.

\section{Investigating coactivation of cells unravels network characteristics related to maturity of olfactory sensory neurons}

We generated cell specific coactivation networks, by weighting edges on how unusually they appear in the dataset. Specifically, we included edges between two coactive genes if they appeared in less than $1 \%$ of the cell population, effectively weighting towards coactivation events that are rarely present than prevalent coactivating events. Upon examining some of these individual cell networks, it appeared that some had a very clear hub-partner topology, characterized by many partners leading to one or two nodes and no other connections (Fig. 5 bottom row), and others were more dense in the number of connections between different nodes (Fig. 5 top row). This suggests that for some cells, a single gene is uniquely activating, and thus coactivation occurs with the other genes that may be active in more cells, whereas for others there are a number of genes appearing uniquely, lending itself to a more densely connected network. In order to identify possible reasons for these different topologies, we considered comparing the centralization measures between 


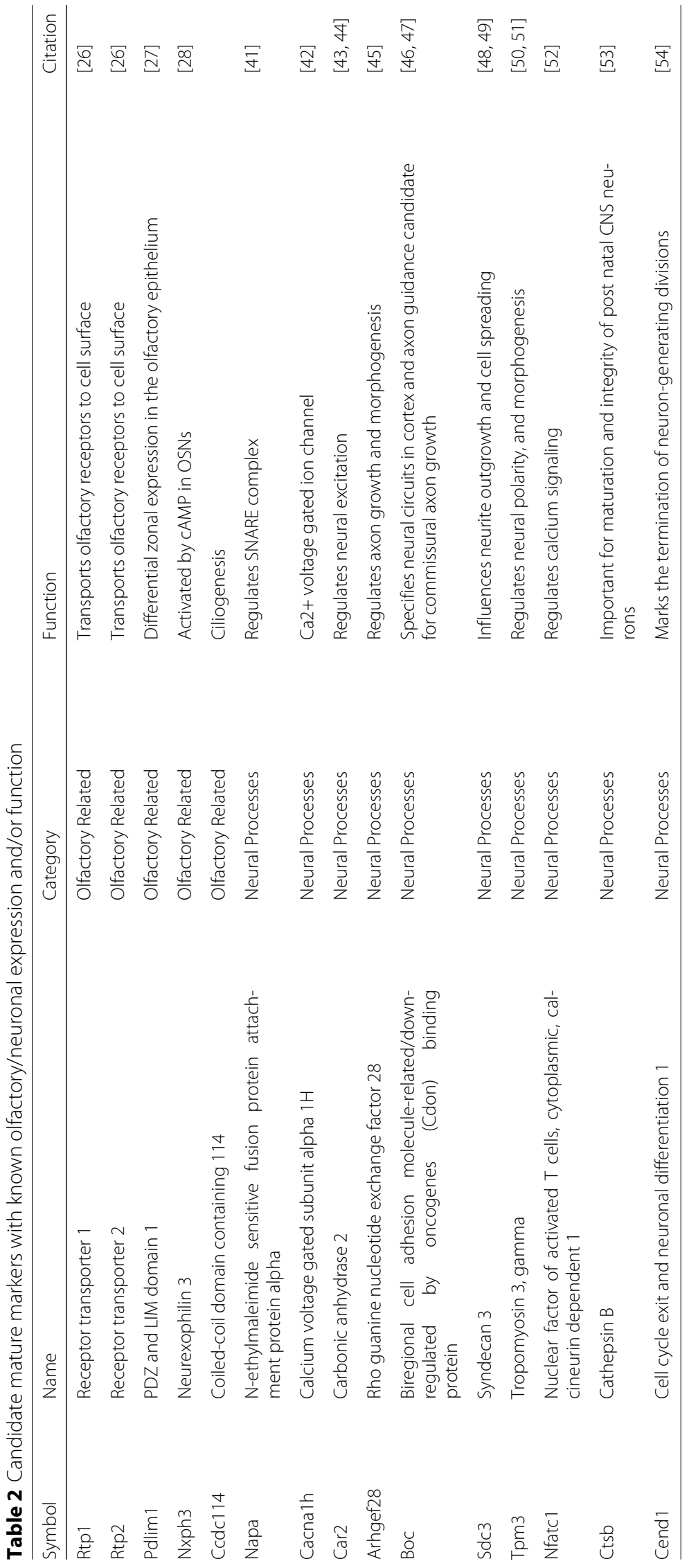




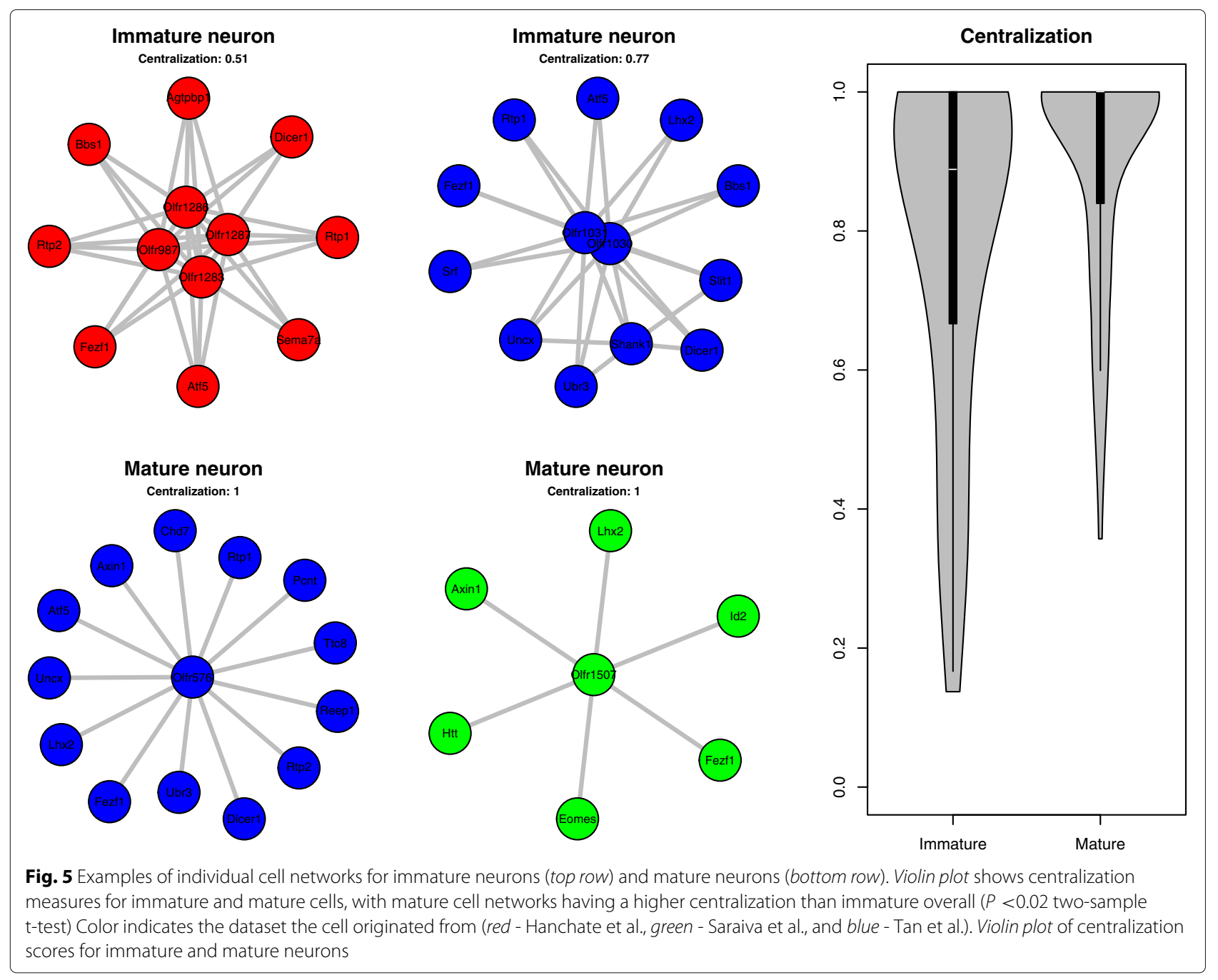

cells that are mature OSNs and immature OSNs. Centralization is a measure of how central connections are towards some nodes, and are higher in networks with hub-partner topology [29]. As described earlier, we identified immature OSNs as those cells that were active for the gene GAP43 and inactive for OMP, and mature OSNs as those cells that were active for $O M P$ and inactive for GAP43. We considered only non-trivial networks with at least 5 nodes, resulting in 111 individual mature cell networks and 39 individual immature cell networks. We found that mature cell networks tend to be more central than immature cell networks $(P<0.01$, two-sided two-sample t-test). To ensure robustness of this result to choice of thresholds, we also compared networks with only edges appearing in less than $0.5,1,2,3,4$, and $5 \%$ of the cell population. In all cases we observed a significant difference in centralization between the two groups ( $P$-values $0.012,0.013,0.0032,0.003,0.0011$, and 0.00046 respectively, two-sided two-sample t-test). Some representative cells from these groups are shown in
Fig. 5. The entire set of non-trivial networks is shown in Additional file 1.

\section{Discussion and conclusions}

In this paper we propose a method to identify transcriptionally active (highly expressed) gene patterns in single cell RNA-Seq data. This was achieved by employing a gamma-normal mixture modeling approach. This gene expression classification further enabled key observations in neuronal cell quality control, and facilitated examination of maturity markers with improved identification in combining datasets.

There has been some discussion as to what causes the apparent bimodal distribution of scRNA-Seq data, including attributing these highly expressed genes to transcriptional bursting [14], referring to very rapid production of RNA occurring in bursts, owing to the stochastic nature of transcription in the cell. Indeed, transcriptional bursting has been explored both theoretically [30], within cellline studies [31], and in the context of scRNA-Seq data 
[13]. Our mixture modeling framework enables identification of genes for which the cell is possibly undergoing transcriptional bursting or is highly expressed, as those that were deemed 'active' throughout this paper, and thus potentially can be used to analyze bursting states given a suitable experimental protocol.

Potential limitations of the method introduced in this paper is the treatment of zero counts. In the case where there are many false positive reads, that is, reads mapped to a gene when in fact there is no underlying transcription occurring, the error may propagate and cells be classified as lowly or less likely highly expressed. A strategy for dealing with this issue may be to incorporate a third component into the gamma-normal mixture model, where the third component has a very high probability density at zero, but also incorporates a non-zero probability for non-zero values. Of course, this requires that the proportion of non-zero values can be estimated somehow. However, in this paper our key observations stemmed from focusing specifically on active genes, and potential issues associated with false positive reads are negligible in this setting.

Additional methodological developments are needed for datasets and genes that do not have a clear bimodal distribution of expression values. These are cells with very little to no highly active genes and did not have enough cells to accurately fit the gamma-normal mixture model, e.g. Fig. 2 for OLFR726. Given a suitable continuous normalization approach, this issue of not enough cells can be ameliorated by simply combining the cells into one large merged dataset. This of course is dependent on a reliable cross-dataset normalization strategy. Methods on batch correction [32] and normalization of bulk RNA-Seq [33-35] data exist, but it is not yet clear how applicable these approaches are given the unique characteristics of scRNASeq such as the abundance of zero values, with strides in effective normalization of scRNA-Seq data actively developing [36].

Using methods to identify active genes and coactive gene pairs within cells, we have been able to identify gene markers for olfactory sensory neuron maturity across multiple datasets, and to observe characteristics of cellspecific coactivation networks weighted by uniqueness. This unique way of exploring single cell RNA-Seq data has enabled interesting observations and future applications to other types of single cell RNA-Seq will be of interest.

\section{Additional files}

Additional file 1: Figure S1. Violin plots of log2CPM values stratified by classification of 1 (lowly expressed), 2 (highly expressed) and NA (not enough data to classify) before (left of dashed line) and after (right of dashed line) employing contextualization of genes, resulting in better separation of log2CPM values between classes 1 and 2, and removal of missing values from the method. (PDF $50.5 \mathrm{~KB}$ )

Additional file 2: Figure S2. Scatterplots of total read depth versus number of non-zero log2CPM values (top left) and (middle left) number of active genes using all genes. Boxplots (top right, middle right, respectively) are of the number of non-zero log2CPM values and number of active genes using all genes respectively, split by dataset. The last boxplot (bottom left) is of total read depth of cells from various datasets. Unsurprisingly, we observe some relationship between total read depth and number of non-zero genes (top left), which is slightly diminished when comparing total read depth to the number of active genes (middle left) for datasets with lower total read depth. (PDF 455 KB)

Additional file 3: Figure S3. Scatterplot of number of olfactory genes with non-zero values against number of olfactory genes classified as active (highly expressed). The gray solid line is the diagonal line and other dotted lines are fitted lines for each dataset. (PDF 225 KB)

Additional file 4: Table S1. This $x \mid s x$ file contains candidate mature markers with unknown olfactory/neuronal expression and/or function. (XLSX 8.15 KB)

Additional file 5: Cell-specific network graphs. This pdf file contains cell name (SRA or equivalent ID), visualization of the cell uniqueness network, its centralization score as well as its classification as a 'mature', 'immature' or 'unsure' of maturity cell, for all 211 non-trivial cell networks. (PDF 498 KB)

\section{Abbreviations}

CPM: Counts per million; EM: Expectation maximization; ENA: European nucleotide archive; FISH: Fluorescence in situ hybridization; GEO: Gene expression omnibus; GO gene ontology; OSN: Olfactory sensory neuron; RPKM: Reads per kilobase per million; sCRNA-Seq: Single cell RNA-Sequencing; SRA: Sequence read archive

\section{Acknowledgments}

The authors thank all their colleagues, particularly at The University of Sydney, School of Mathematics and Statistics, for support and intellectual engagement.

\section{Declarations}

This article has been published as part of BMC Systems Biology Volume 10 Supplement 5, 2016. 15th International Conference On Bioinformatics (INCOB 2016): systems biology. The full contents of the supplement are available online http://bmcsystbiol.biomedcentral.com/articles/supplements/volume10-supplement-5.

\section{Funding}

The following sources of funding for each author, and for the manuscript preparation, are gratefully acknowledged: Australian Postgraduate Award (SG); CSIRO OCE Top-Up Scholarship (SG); Australian Research Council Future Fellowship grant \#FT0991918 and DP grant \#130100488 (JYHY); Australian Research Council DECRA Fellowship \#DE130101670 (JTO); Cornell University College of Veterinary Medicine Research Grant Program (DML) and Training Grant in Vertebrate Developmental Genomics (NIH T32HD057854) from the National Institutes of Health (AJB). Publication charges were funded by the Australian Research Council (Future Fellowship grant \#FT0991918 and DP grant \#130100488 (JYHY). The funding source had no role in the study design; in the collection, analysis, and interpretation of data; in the writing of the manuscript; and in the decision to submit the manuscript for publication.

\section{Availability of data and materials}

The data supporting the results of this article have been previously described and are available in the GEO repository under accession IDs GSE70844, GSE75413, GSE63576, GSE52525, PRJEB4014, PRJEB8101, PRJEB4461, SRP065920, GSE71585, GSE59739, and GSE60361. Other data supporting the conclusions of this article are included and cited within the article and its additional files.

\section{Authors' contributions}

The study was conceived by JYHY and DML. SG carried out the analysis in R, supervised by JYHY. JTO and SG derived the EM algorithm. AJB performed biological analysis, supervised by DML. All authors read and critically analyzed 
the manuscript prior to submission. All authors read and approved the final manuscript.

\section{Competing interests}

The authors declare that they have no competing interests.

\section{Consent for publication}

Not applicable.

\section{Ethics approval and consent to participate}

Not applicable.

\section{Author details}

${ }^{1}$ School of Mathematics and Statistics, The University of Sydney, Eastern Avenue, Camperdown, NSW, 2006, Australia. ${ }^{2}$ Department of Biomedical Sciences, Cornell University, Ithaca, NY, 14853, USA. ${ }^{3}$ ARC Centre of Excellence for Mathematical \& Statistical Frontiers, University of Melbourne, Parkville VIC 3010, Australia.

\section{Published: 5 December 2016}

\section{References}

1. Saliba AE, Westermann AJ, Gorski SA, Vogel J. Single-cell RNA-seq: advances and future challenges. Nucleic Acids Res. 2014;42(14):8845-60. doi:10.1093/nar/gku555.

2. Scholz P, Kalbe B, Jansen F, Altmueller J, Becker C, Mohrhardt J, Schreiner B, Gisselmann G, Hatt H, Osterloh S. Transcriptome Analysis of Murine Olfactory Sensory Neurons during Development Using Single Cell RNA-Seq. Chem Senses. 2016;41(4):313-23. doi:10.1093/chemse/bjw003.

3. Macosko EZ, Basu A, Satija R, Nemesh J, Shekhar K, Goldman M, Tirosh I, Bialas AR, Kamitaki N, Martersteck EM, Trombetta JJ, Weitz DA, Sanes JR, Shalek AK, Regev A, McCarroll SA. Highly Parallel Genome-wide Expression Profiling of Individual Cells Using Nanoliter Droplets. Cell. 2015;161(5):1202-14. doi:10.1016/j.cell.2015.05.002.

4. Tan L, Li Q, Xie XS. Olfactory sensory neurons transiently express multiple olfactory receptors during development. Mol Syst Biol. 2015;11(12):844-4. doi:10.15252/msb.20156639.

5. Gu J, Du Q, Wang X, Yu P, Lin W. Sphinx: modeling transcriptional heterogeneity in single-cell rna-seq. bioRxiv. 2015. doi:10.1101/027870.

6. Ntranos V, Kamath GM, Zhang JM, Pachter L, Tse DN. Fast and accurate single-cell RNA-seq analysis by clustering of transcript-compatibility counts. Genome Biol. 2016;17(1):112. doi:10.1186/s13059-016-0970-8.

7. Diaz A, Liu SJ, Sandoval C, Pollen A, Nowakowski TJ, Lim DA, Kriegstein A. SCell: integrated analysis of single-cell RNA-seq data. Bioinformatics. 2016;32(14):2219-20. doi:10.1093/bioinformatics/btw201.

8. Zurauskiene J, Yau C. pcaReduce: hierarchical clustering of single cell transcriptional profiles. BMC Bioinforma. 2016;17(1):140. doi:10.1186/s12859-016-0984-y.

9. Xu C, Su Z. Identification of cell types from single-cell transcriptomes using a novel clustering method. Bioinformatics. 2015;31(12):1974-80 doi:10.1093/bioinformatics/btv088.

10. Kharchenko PV, Silberstein L, Scadden DT. Bayesian approach to single-cell differential expression analysis,. Nat Methods. 2014;11(7): 740-2. doi:10.1038/nmeth.2967.

11. Shalek AK, Satija R, Adiconis X, Gertner RS, Gaublomme JT, Raychowdhury R, Schwartz S, Yosef N, Malboeuf C, Lu D, Trombetta JJ, Gennert D, Gnirke A, Goren A, Hacohen N, Levin JZ, Park H, Regev A. Single-cell transcriptomics reveals bimodality in expression and splicing in immune cells,. Nature. 2013;498(7453):236-40. doi:10.1038/nature12172.

12. McDavid A, Dennis L, Danaher P, Finak G, Krouse M, Wang A, Webster P, Beechem J, Gottardo R. Modeling Bi-modality Improves Characterization of Cell Cycle on Gene Expression in Single Cells. PLoS Comput Biol. 2014;10(7):. doi:10.1371/journal.pcbi.1003696.

13. Kim JK, Marioni JC. Inferring the kinetics of stochastic gene expression from single-cell RNA-sequencing data. Genome Biol. 2013;14(1):7. doi:10.1186/gb-2013-14-1-r7.

14. Suter DM, Molina N, Gatfield D, Schneider K, Schibler U, Naef F. Mammalian Genes Are Transcribed with Widely Different Bursting Kinetics. Science. 2011;332(6028):472-4. doi:10.1126/science.1198817.
15. Saraiva LR, Ibarra-Soria X, Khan M, Omura M, Scialdone A, Mombaerts $P$, Marioni JC, Logan DW. Hierarchical deconstruction of mouse olfactory sensory neurons: from whole mucosa to single-cell RNA-seq. Sci Rep. 2015;5:18178. doi:10.1038/srep18178.

16. Dobin A, Davis Ca, Schlesinger F, Drenkow J, Zaleski C, Jha S, Batut P, Chaisson M, Gingeras TR. STAR: ultrafast universal RNA-seq aligner. Bioinformatics. 2013;29(1):15-21. doi:10.1093/bioinformatics/bts635.

17. Li H, Handsaker B, Wysoker A, Fennell T, Ruan J, Homer N, Marth G, Abecasis G, Durbin R. The Sequence Alignment/Map format and SAMtools. Bioinformatics. 2009;25(16):2078-9. doi:10.1093/bioinformatics/btp352.

18. Anders S, Pyl PT, Huber W. HTSeq-a Python framework to work with high-throughput sequencing data. Bioinformatics. 2015;31(2):166-9. doi:10.1093/bioinformatics/btu638.

19. Kream RM, Margolis FL. Olfactory marker protein: Turnover and transport in normal and regenerating neurons. J Neurosci. 1984;4(3):868-79.

20. Verhaagen J, Oestreicher AB, Grillo M, Khew-Goodall YS, Gispen WH, Margolis FL. Neuroplasticity in the olfactory system: differential effects of central and peripheral lesions of the primary olfactory pathway on the expression of B-50/GAP43 and the olfactory marker protein. J Neurosci Res. 1990;26(1):31-44. doi:10.1002/jnr.490260105.

21. Margolis FL, Verhaagen J, Biffo S, Huang FL, Grillo M. Regulation of gene expression in the olfactory neuroepithelium: a neurogenetic matrix. Prog Brain Res. 1991;89:97-122.

22. Nickell MD, Breheny P, Stromberg AJ, Mcclintock TS. Genomics of mature and immature olfactory sensory neurons. J Comp Neurol. 2012;520(12):2608-29. doi:10.1002/cne.23052.

23. Li CL, Li KC, Wu D, Chen Y, Luo H, Zhao JR, Wang SS, Sun MM, Lu YJ, Zhong YQ, Hu XY, Hou R, Zhou BB, Bao L, Xiao HS, Zhang X. Somatosensory neuron types identified by high-coverage single-cell RNA-sequencing and functional heterogeneity. Cell Res. 20151-20. doi:10.1038/cr.2015.149.

24. Lovatt D, Ruble BK, Lee J, Dueck H, Kim TK, Fisher S, Francis C, Spaethling JM, Wolf Ja, Grady MS, Ulyanova AV, Yeldell SB, Griepenburg JC, Buckley PT, Kim J, Sul JY, Dmochowski IJ, Eberwine J. Transcriptome in vivo analysis (TIVA) of spatially defined single cells in live tissue. Nat Methods. 2014;11(2):190-6. doi:10.1038/nmeth.2804.

25. Hanchate NK, Kondoh K, Lu Z, Kuang D, Ye X, Qiu X, Pachter L, Trapnell $C$, Buck LB. Single-cell transcriptomics reveals receptor transformations during olfactory neurogenesis. Science. 2015;350(6265):1251-5. doi:10.1126/science.aad2456.

26. Saito H, Kubota M, Roberts RW, Chi Q, Matsunami H. RTP family members induce functional expression of mammalian odorant receptors,. Cell. 2004;119(5):679-91. doi:10.1016/j.cell.2004.11.021.

27. Tietjen I, Rihel J, Dulac CG. Single-cell transcriptional profiles and spatial patterning of the mammalian olfactory epithelium. Int J Dev Biol. 2005;49(2-3):201-7. doi:10.1387/ijdb.041939it.

28. Imai T, Suzuki M, Sakano H. Odorant Receptor-Derived cAMP Signals Direct Axonal Targeting. Science. 2006;314(5799):657-61. doi:10.1126/science.1131794.

29. Freeman LC. Centrality in social networks conceptual clarification. Soc Networks. 1978;1(3):215-39. doi:10.1016/0378-8733(78)90021-7.

30. Kumar N, Singh A, Kulkarni RV. Transcriptional Bursting in Gene Expression: Analytical Results for General Stochastic Models. PLoS Comput Biol. 2015;11(10):1-22. doi:10.1371/journal.pcbi.1004292. arXiv: $1412.8634 \mathrm{v} 1$

31. Raj A, Peskin CS, Tranchina D, Vargas DY, Tyagi S. Stochastic mRNA synthesis in mammalian cells. PLoS Biology. 2006;4(10):1707-1719. doi:10.1371/journal.pbio.0040309.

32. Johnson WE, Li C, Rabinovic A. Adjusting batch effects in microarray expression data using empirical Bayes methods. Biostatistics. 2007;8(1): 118-27. doi:10.1093/biostatistics/kxj037.

33. Risso D, Ngai J, Speed TP, Dudoit S. Normalization of RNA-seq data using factor analysis of control genes or samples (RUVSeq). Nat Biotechnol. 2014;32(9):896-902. doi:10.1038/nbt.2931.

34. Love MI, Huber W, Anders S. Moderated estimation of fold change and dispersion for RNA-seq data with DESeq2. Genome Biol. 2014;15(12):550. doi:10.1186/s13059-014-0550-8.

35. Robinson MD, Oshlack A. A scaling normalization method for differential expression analysis of RNA-seq data. Genome Biol. 2010;11(3):25. doi:10.1186/gb-2010-11-3-r25. 
36. L. Lun AT, Bach K, Marioni JC. Pooling across cells to normalize single-cell RNA sequencing data with many zero counts. Genome Biol. 2016;17(1): 75. doi:10.1186/s13059-016-0947-7.

37. Fuzik J, Zeisel A, Máté Z, Calvigioni D, Yanagawa Y, Szabó G, Linnarsson S, Harkany T. Integration of electrophysiological recordings with single-cell RNA-seq data identifies neuronal subtypes. Nat Biotechnol. 2015;34(2):175-83. doi:10.1038/nbt.3443.

38. Tasic B, Menon V, Nguyen TN, Kim TK, Jarsky T, Yao Z, Levi B, Gray LT, Sorensen SA, Dolbeare T, Bertagnolli D, Goldy J, Shapovalova N, Parry S, Lee C, Smith K, Bernard A, Madisen L, Sunkin SM, Hawrylycz M, Koch C, Zeng $\mathrm{H}$. Adult mouse cortical cell taxonomy revealed by single cell transcriptomics. Nat Neurosci. 2016;19(2):335-46. doi:10.1038/nn.4216.

39. Usoskin D, Furlan A, Islam S, Abdo H, Lönnerberg P, Lou D, Hjerling-Leffler J, Haeggström J, Kharchenko O, Kharchenko PV, Linnarsson S, Ernfors P. Unbiased classification of sensory neuron types by large-scale single-cell RNA sequencing. Nat Neurosci. 2014;18(1): 145-53. doi:10.1038/nn.3881.

40. Zeisel A, Manchado ABM, Codeluppi S, Lonnerberg P, La Manno G, Jureus A, Marques S, Munguba H, He L, Betsholtz C, Rolny C, Castelo-Branco G, Hjerling-Leffler J, Linnarsson S. Cell types in the mouse cortex and hippocampus revealed by single-cell RNA-seq. Science. 2015;347(6226):1138-42. doi:10.1126/science.aaa1934. 9809069v1.

41. Söllner T, Whiteheart SW, Brunner M, Erdjument-Bromage H, Geromanos $\mathrm{S}$, Tempst $\mathrm{P}$, Rothman JE. SNAP receptors implicated in vesicle targeting and fusion. Nature. 1993;362(6418):318-24. doi:10.1038/362318a0.

42. Maue RA. Patch-clamp studies of isolated mouse olfactory receptor neurons. J Gen Physiol. 1987;90(1):95-125. doi:10.1085/jgp.90.1.95.

43. Ruusuvuori E, Huebner AK, Kirilkin I, Yukin AY, Blaesse P, Helmy M, Jung Kang H, El Muayed M, Christopher Hennings J, Voipio J, Šestan N, Hübner CA, Kaila K. Neuronal carbonic anhydrase VII provides GABAergic excitatory drive to exacerbate febrile seizures. EMBO J. 2013;32(16): 2275-86. doi:10.1038/emboj.2013.160.

44. Ruusuvuori E, Kaila K. Carbonic anhydrases and brain pH in the control of neuronal excitability. Sub-cellular Biochem. 2014;75:271-90. doi:10.1007/978-94-007-7359-2_14.

45. Mulinari S, Häcker U. Rho-guanine nucleotide exchange factors during development. Small GTPases. 2010;1 (1):28-43. doi:10.4161/sgtp.1.1.12672.

46. Okada A, Charron F, Morin S, Shin DS, Wong K, Fabre PJ, Tessier-Lavigne M, McConnell SK. Boc is a receptor for sonic hedgehog in the guidance of commissural axons. Nature. 2006;444(7117):369-73. doi:10.1038/nature05246.

47. Harwell CC, Parker PRL, Gee SM, Okada A, McConnell SK, Kreitzer AC, Kriegstein AR. Sonic Hedgehog Expression in Corticofugal Projection Neurons Directs Cortical Microcircuit Formation. Neuron. 2012;73(6): 1116-26. doi:10.1016/j.neuron.2012.02.009.

48. Toba Y, Horie M, Sango K, Tokashiki A, Matsui F, Oohira A, Kawano H. Expression and immunohistochemical localization of heparan sulphate proteoglycan $\mathrm{N}$-syndecan in the migratory pathway from the rat olfactory placode. Eur J NeuroSci. 2002;15(9):1461-73. doi:10.1046/j.1460-9568.2002.01983.x.

49. Bespalov MM, Sidorova YA, Tumova S, Ahonen-Bishopp A, Magalhães AC, Kulesskiy E, Paveliev M, Rivera C, Rauvala H, Saarma M. Heparan sulfate proteoglycan syndecan-3 is a novel receptor for GDNF, neurturin, and artemin. J Cell Biol. 2011;192(1):153-69. doi:10.1083/jcb.201009136.

50. Dufour C, Weinberger RP, Gunning P. Tropomyosin isoform diversity and neuronal morphogenesis. Immunol Cell Biol. 1998;76(5):424-9. doi:10.1046/j.1440-1711.1998.00765.x.

51. Schevzov G. Specific Features of Neuronal Size and Shape Are Regulated by Tropomyosin Isoforms. Mol Biol Cell. 2005;16(7):3425-37. doi:10.1091/mbc.E04-10-0951.

52. Graef IA, Mermelstein PG, Stankunas K, Neilson JR, Deisseroth K, Tsien RW, Crabtree GR. L-type calcium channels and GSK-3 regulate the activity of NF-ATc4 in hippocampal neurons. Nature. 1999;401 (6754):703-8. doi:10.1038/44378.

53. Felbor U, Kessler B, Mothes W, Goebel HH, Ploegh HL, Bronson RT, Olsen BR. Neuronal loss and brain atrophy in mice lacking cathepsins B and L. Proc Natl Acad Sci. 2002;99(12):7883-8. doi:10.1073/pnas.112632299.

54. Politis PK, Makri G, Thomaidou D, Geissen M, Rohrer H, Matsas R. BM88/CEND1 coordinates cell cycle exit and differentiation of neuronal precursors. Proc Natl Acad Sci. 2007;104(45):17861-6. doi:10.1073/pnas.0610973104.

\section{Submit your next manuscript to BioMed Central and we will help you at every step:}

- We accept pre-submission inquiries

- Our selector tool helps you to find the most relevant journal

- We provide round the clock customer support

- Convenient online submission

- Thorough peer review

- Inclusion in PubMed and all major indexing services

- Maximum visibility for your research

Submit your manuscript at www.biomedcentral.com/submit 